\title{
A PROBLEM OF WIENER AND THE FAILURE OF A PRINCIPLE FOR FOURIER SERIES WITH POSITIVE COEFFICIENTS
}

\author{
STEPHEN WAINGER ${ }^{1}$
}

Wiener proved the following theorem [1].

Theorem A. Let

$$
F(z)=\sum_{n=0}^{\infty} a_{n} z^{n}
$$

be analytic in $|z|<1$, and assume $a_{n} \geqq 0$. Furthermore, assume for some $\delta>0$,

$$
\sup _{0 \leqq r<1} \int_{-\delta}^{\delta}\left|F\left(r e^{i \theta}\right)\right|^{2} d \theta \leqq M<\infty .
$$

Then

$$
\sup _{0 \leqq r<1} \int_{-\pi}^{\pi}\left|F\left(r e^{i \theta}\right)\right|^{2} d \theta \leqq N<\infty .
$$

Wiener then asked if the analogue of his theorem were true for $H^{1}$ [1]. (That is, whether the square in the integrals of Theorem A may be replaced by the first power.) This question has added interest now because Boas [2], [3] has shown that if a function with positive Fourier series coefficients is "good" in a neighborhood of zero, then the function is "good" everywhere for several senses of the word "good." In this note we point out that a result of Hardy implies a negative answer to Wiener's question, and at the same time gives an example of a trigonometric series with positive coefficients whose behaviour is good near the origin but not everywhere.

Theorem B. Let $0<p<2$. Then there exists

$$
F(z)=\sum_{n=1}^{\infty} a_{n} z^{n}
$$

analytic in $|z|<1$ with $a_{n} \geqq 0$ and $\delta>0$ such that

$$
\sup _{r \leqq 1} \int_{-\delta}^{\delta}\left|F\left(r e^{i \theta}\right)\right|^{p} d \theta \leqq M<\infty ;
$$

Received by the editors October 2, 1967.

1 Supported in part by NSF Grant GP-6764. 


$$
\sup _{r<1} \int_{-\pi}^{\pi}\left|F\left(r e^{i \theta}\right)\right| p d \theta=\infty .
$$

REMARK. $\delta$ may be chosen to be any number less than $\pi$.

ProOF OF TheOREM B. We take $a_{n}=n^{-\gamma}\left(2+\cos \lambda n \cos n^{\alpha}\right)$, with $\pi>\lambda>\delta$. Let

$$
h(r, \theta)=\sum_{n=1}^{\infty} r^{n} n^{-\gamma} e^{i n \theta} .
$$

As $\theta \rightarrow 0, h(\theta) \simeq k_{\gamma} \theta^{-1+\gamma}$ with $k_{\gamma} \neq 0$, where $h(\theta)=\lim _{r \rightarrow 1} h(r, \theta)$. Furthermore as one would then expect

$$
\sup _{0 \leqq r<1} \int_{-\pi}^{\pi}|h(r, \theta)|^{p} d \theta \leqq M<\infty, \quad \text { for } p(1-\gamma)<1 .
$$

See $[5$, Chapter 5$]$. Let

$$
g(r, \theta)=\sum_{n=1}^{\infty} r^{n} n^{-\gamma} \exp \left(i n^{\alpha}\right) \exp (i n \theta), \quad 0<\alpha<1 .
$$

Hardy showed [4] $g(\theta)=\lim _{r \rightarrow 1} g(r, \theta)$ exists for $\theta \neq 0$, and

$$
|g(\theta)| \simeq k(\alpha, \gamma)|\theta|^{-(1-\gamma-\alpha / 2) /(1-\alpha)}
$$

with $k(\alpha, \gamma) \neq 0$, as $\theta$ tends to zero from the left, is bounded as $\theta$ tends to zero from the right, and is otherwise continuous in $[-\pi, \pi]$. Also, $g(r, \theta)$ is bounded in any portion of the unit circle lying above a line $y=\beta(x-1)$. ( $x$ and $y$ are here the usual rectangular coordinates and $-\infty<\beta<\infty$. The bound of course depends on $\beta$.) $F\left(r e^{i \theta}\right)$ is made up of two parts, one having a singularity like $h(r, \theta)$ at $r=1, \theta=0$; the other having singularities like that of $g(r, \theta)$ except translated to $\theta=+\lambda$ and $\theta=-\lambda$.

We choose any $\gamma$ with $1 / 2>\gamma>1-1 / p$. Then $\|h(r, \theta)\|_{p}$ will be bounded uniformly in $r<1$ and $F\left(r e^{i \theta}\right)$ will satisfy the hypothesis (1). But now, by choosing $\alpha$ sufficiently close to 1 , we can make $|g(\theta)|$ $\simeq c \theta^{-l}$ for arbitrarily large $l(c \neq 0)$. Thus $F(\theta)$ is not in $L^{p}$. Hence

$$
\sup _{r<1} \int_{-\pi}^{\pi}\left|F\left(r e^{i \theta}\right)\right|^{p} d \theta=\infty .
$$

See [5, Chapter 7].

REMARK. The analogue of Wiener's Theorem holds for $p=2 k, k$ $=1,2, \cdots$. (That is, Theorem $B$ is false for these values. This follows from Wiener's Theorem. What happens for arbitrary $p>2$ seems an interesting problem.) 


\section{REFERENCES}

1. R. P. Boas, Entire functions, Academic Press, New York, 1964; pp. 242, 250.

2. - Fourier series with positive coefficients, J. Math. Anal. Appl. 17 (1967), $463-483$.

3. - Integrability theorems for trigonometric transforms, Springer-Verlag, Berlin, 1967.

4. G. H. Hardy, A theorem concerning Taylor's series, Quart. J. Pure Appl. Math. 44 (1913), 147-160.

5. A. Zygmund, Trigonometric series, Vol. I, Cambridge Univ. Press, New York, 1959.

UNIVERSITY OF WISCONSIN 